\title{
Comparação entre as Terminologias Padronizadas por Baden e Walker e pela ICS para o Prolapso Pélvico Feminino
}

\author{
Comparison Between Baden and Walker Classification Systems and ICS \\ Standardization of Terminology of Female Pelvic Organ Prolapse \\ Leonardo Robson Pinheiro Sobreira Bezerra, Emerson de Oliveira, \\ Maria Augusta Tezelli Bortolini, Maria Gorete Hamerski, Edmund Chada Baracat, \\ Marair Gracio Ferreira Sartori, Manoel João Bastista Castello Girão
}

\section{RESUM0}

Objetivo: comparar a classificação de Baden e Walker (BW) para o prolapso pélvico feminino e a preconizada pela Sociedade Internacional de Continência (ICS).

Métodos: em trabalho retrospectivo foram analisadas as informações sobre 101 pacientes atendidas no setor de Uroginecologia e Cirurgia Vaginal do Departamento de Ginecologia da UNIFESP/EPM durante investigação uroginecológica. As pacientes foram selecionadas a partir da revisão do prontuário médico, onde foram identificadas aquelas que submeteramse a exame padronizado pela ICS a fim de quantificar o prolapso pélvico feminino. Conforme preconiza a ICS, o prolapso foi analisado por um sistema padrão de referência que relaciona a carúncula himenal (ponto fixo) à posição anatômica de seis pontos definidos: 2 na parede vaginal anterior, 2 no ápice vaginal e 2 na parede vaginal posterior. A máxima protrusão do prolapso foi visualizada e registrada durante a manobra de Valsalva solicitada à paciente. Realizou-se a medida do ponto mais externo do prolapso (pontos $\mathrm{Ba}, \mathrm{Bp}$ e C) comparando-o com a classificação de BW. A medida adotada para avaliar a concordância entre as duas terminologias foi a estatistica kappa.

Resultados: observou-se correspondência de 100\% somente para o prolapso de parede vaginal posterior estádio IV (1 paciente) e para o prolapso uterino estádio zero (29 pacientes), segundo Baden e Walker, com retocele severa e ausência de prolapso, respectivamente. Para os três tipos de prolapsos examinados, os valores da estatística kappa estavam abaixo de 0,4, indicando fraca concordância entre as duas terminologias. Concluímos que existe uma ampla variação nas medidas do ponto mais externo do prolapso ao se realizar a classificação de BW. Para um determinado grau de prolapso na classificação de BW encontramos mais de um estádio na classificação da ICS.

Conclusões: existe fraca concordância entre as classificações de Baden e Walker e a da Sociedade Internacional de Continência para as distopias genitais.

PALAVRAS-CHAVE: Prolapso genital. Assoalho pélvico. Incontinência urinária.

Universidade Federal de São Paulo - EPM/UNIFESP; Departamento de Ginecologia da Escola Paulista de Medicina

Correspondência:

Leonardo Robson Pinheiro Sobreira Bezerra

Setor de Uroginecologia e Cirurgia Vaginal, Departamento de Ginecologia da Universidade Federal de São Paulo

Rua dos Otonis 567 - Vila Clementino

04025-001 - São Paulo - SP

Fone (Fax): (11) 5084-2514

e-mail: leosobreira@uol.com.br
Introdução

Define-se como prolapso genital o deslocamento das vísceras pélvicas no sentido caudal, em direção ao hiato genital. Decorre do desequilíbrio entre as forças que mantêm os órgãos pélvicos em sua posição normal e aquelas que tendem a impeli-los para fora da pelve ${ }^{1}$. A 
Sociedade Internacional de Continência ${ }^{2}$ (ICS), por sua vez, define como prolapso genital o descenço da parede vaginal anterior e/ou posterior assim como do ápice da vagina (cérvix/útero) ou da cúpula vaginal após histerectomia. Constitui achado relativamente comum. Nos Estados Unidos da América relata-se taxa de prevalência de 2,1 por 1000 mulheres $^{3}$. Embora não ocasione risco de morte, pode afetar a qualidade da vida de muitas mulheres. Além disso, é responsável pela cifra de 400.000 procedimentos cirúrgicos anualmente nos Estados Unidos da América ${ }^{4}$. O prolapso genital provoca sintomatologia variada, em geral relacionada aos vários compartimentos anatômicos afetados ${ }^{5}$. Nota-se aumento significante das queixas em conformidade com o avanço do estádio do prolapso. No estudo de Swift et al. ${ }^{6}$, somente $2 \%$ das pacientes classificadas no estádio 2 tinham queixa importante de "bola na vagina", aumentando para $78 \%$ naquelas no estádio 3.

Parece clara, portanto, a necessidade de se registrarem os achados de exame físico em um padrão reprodutível e inteligivel com o objetivo de se estabelecerem critérios de gravidade para melhor intervenção terapêutica. Em vista disso, vários autores vem tentando codificar a classificação do prolapso pélvico nas últimas décadas, valendo-se de parâmetros de gravidade progressiva em relação a referenciais fixos, ora o intróito vaginal, ora o terço médio ou distal da vagina.

A classificação de Baden e Walker modifica$\mathrm{da}^{7,8}$ é a de uso mais difundido em nosso meio. São utilizadas denominações distintas para os diferentes compartimentos. A distopia da uretra e da bexiga, uretro ou cistocele, seria classificada nos seguintes graus: $0,1^{\circ}, 2^{\circ}, 3^{\circ}$ e $4^{\circ}$ grau. A distopia da parede vaginal posterior, ou retocele, classificada de acordo com graus de gravidade: leve, moderada e grave. Já o prolapso uterino é classificado em $1^{\circ}, 2^{\circ}$ e $3^{\circ}$ graus, com o colo pinçado e tracionado durante o exame genital ${ }^{8}$.

Com o objetivo de padronizar a terminologia e a classificação da disfunção do assoalho pélvico, a Sociedade Internacional de Continência, juntamente com a Sociedade de Uroginecologia e a Sociedade Americana de Ginecologia, elaboraram em 1996 nova classificação para avaliar o prolapso genital $^{9,10}$. Trata-se de sistema descritivo que contém vários componentes a serem medidos.

O objetivo deste estudo foi comparar o estadiamento da distopia genital proposto por esta nova terminologia da $\mathrm{ICS}^{2}$ com o sistema de classificação de Baden e Walker ${ }^{7,8}$, procurando avaliar discrepâncias e imprecisões.

\section{Pacientes e Métodos}

Realizamos estudo retrospectivo no qual foram incluídas 101 pacientes atendidas no Ambulatório de Uroginecologia e Cirurgia Vaginal da Unifesp/EPM, no período de junho a setembro 2002. As pacientes foram selecionadas a partir da revisão do prontuário médico e apresentavam como diagnóstico incontinência urinária e/ou prolapso genital.

A média de idade foi de 51,2 anos (19 a 82), sendo que 48 (47\%) encontravam-se no menacme e $53(53 \%)$ na pós-menopausa. A paridade média foi de 3,9 filhos (0 a 18).

Pela pesquisa do prontuário foram identificadas as pacientes que submeteram-se a realização do exame padronizado pela ICS a fim de quantificar o prolapso pélvico e, a seguir, comparamos o estádio $\mathrm{ICS}^{2} \mathrm{com}$ a classificação de Baden e Walker ${ }^{7,8}$ para o prolapso pélvico feminino. As pacientes foram examinadas em posição ginecológica, estando a mesa ginecológica com inclinação de 45‥ A máxima protrusão do prolapso foi visualizada e registrada durante a manobra de Valsalva solicitada à paciente. Segundo orientação da padronização da ICS $^{2}$, evitou-se o uso de termos como cistocele, retocele, enterocele ou junção uretrovesical. Portanto, para o propósito da discussão dessa nova terminologia, o prolapso pélvico feminino incluiu o prolapso de parede vaginal anterior (conhecido como cistocele), o prolapso de cúpula vaginal ou uterino e o prolapso de parede vaginal posterior (conhecido como retocele e/ou enterocele).

Conforme preconiza a $\mathrm{ICS}^{2}$, a descrição do prolapso de órgãos pélvicos é feita durante o exame físico da genitália externa e do canal vaginal, bastando apenas espéculo vaginal e histerômetro ou régua. O prolapso deve ser analisado por um sistema padrão de referência que relaciona a carúncula himenal (ponto fixo) à posição anatômica de seis pontos definidos: dois na parede vaginal anterior, dois no ápice vaginal e dois na parede vaginal posterior. Estes pontos são localizados em relação ao plano do hímen, sendo este definido como zero. Serão negativos aqueles que estiverem centímetros acima ou proximais ao hímen e positivos aqueles centímetros abaixo ou distais ao hímen.

Os pontos anteriores são: 1- ponto Aa: localizado na parede vaginal anterior, $3 \mathrm{~cm}$ proximal ao meato externo da uretra, correspondendo à localização aproximada da junção uretro-vesical. Sua posição pode variar de $-3 \mathrm{a}+3 \mathrm{~cm}$; 2 - ponto Ba: é o ponto mais distal de qualquer parte da parede va- 
ginal anterior. Por definição, o ponto Ba está a -3 cm na ausência de prolapso.

Os pontos localizados no ápice da vagina são: 1- ponto C: representa o ponto mais distal da extremidade da cérvix ou do fundo de saco vaginal após histerectomia total; 2- ponto D: representa a localização do fórnix posterior (fundo de saco de Douglas) na mulher com colo uterino. Corresponde à altura em que os ligamentos útero-sacrais se ligam à cérvix proximal posterior. É omitido na ausência de cérvix.

Encontram-se na parede vaginal posterior: 1- ponto Ap: localizado na parede vaginal posterior, $3 \mathrm{~cm}$ proximal ao hímen. Por definição, a variação da posição do ponto Ap em relação ao hímen é de $-3 \mathrm{a}+3 \mathrm{~cm}$; 2 - ponto Bp: representa a posição mais distal de qualquer parte da parede vaginal posterior. Está a $-3 \mathrm{~cm}$ na ausência de prolapso.

Durante a descrição do prolapso pélvico são realizadas outras três medidas: 1- hiato genital (HG): é a medida da linha média do meato externo da uretra à linha média posterior do hímen, 2corpo perineal (CP): é a medida da margem posterior do hiato genital ao ponto médio do orifício anal, e 3- comprimento vaginal total (CVT): é o ponto mais profundo da vagina quando os pontos C e D estão na sua posição normal. Todas as medidas, com exceção do CVT, são realizadas com a paciente fazendo manobra de Valsava.

As medidas podem ser sinaladas como uma seqüência de números respectivamente para os pontos $\mathrm{Aa}, \mathrm{Ba}, \mathrm{C}, \mathrm{D}, \mathrm{Bp}, \mathrm{Ap}, \mathrm{CVT}, \mathrm{HG}, \mathrm{CP}$ ou pode ser usada uma grade de barras $3 \times 3$ para organizálas de forma mais concisa (Tabela 1).

Tabela 1 - Grade de barras $3 \times 3$ usada para sinalar o prolapso pélvico feminino

\begin{tabular}{l|l|l}
$A a$ & $B a$ & $C$ \\
\hline$H G$ & $C P$ & $C V T$ \\
\hline$A p$ & $B p$ & $D$
\end{tabular}

Parede vaginal anterior: Aa: Localização aproximada da junção uretro-vesical; Ba: Ponto mais distal de qualquer parte da parede vaginal anterior.

Ápice da vagina: C: Ponto mais distal da extremidade da cérvix ou do fundo de saco vaginal após histerectomia total; D: Localização do fórnix posterior (fundo de saco de Douglas) na mulher com colo uterino.

Parede vaginal posterior: Ap: Localizado na parede vaginal posterior, $3 \mathrm{~cm}$ proximal ao hímen; Bp: Posição mais distal de qualquer parte da parede vaginal posterior.

Outras medidas: HG: Medida da linha média do meato externo da uretra à linha média posterior do hímen; CP: Medida da margem posterior do hiato genital ao ponto médio do orifício anal; CVT: Ponto mais profundo da vagina quando o ponto $C$ e $D$ estão na sua posição normal.

Por fim, a $\mathrm{ICS}^{2}$ estabeleceu um sistema de estádios para o prolapso pélvico que são sinalados de acordo com a posição mais distal do prolapso quando sua extensão foi completamente demons- trada. Portanto, para que o estádio seja definido, é necessário que sua descrição quantitativa seja primeiro completada.

Os estádios podem ser subagrupados de acordo com a porção do trato reprodutivo baixo que é a parte mais distal do prolapso, pelo uso das seguintes letras: $\mathrm{a}=$ parede vaginal anterior; $\mathrm{p}=$ parede vaginal posterior; $\mathrm{C}=$ fundo de saco vaginal; $\mathrm{Cx}=$ cérvix, e os pontos de medidas já definidos.

Os estádios são os seguintes: a- estádio 0 : não se demonstra prolapso. Os pontos aa, $\mathrm{Ba}, \mathrm{Ap}$, Bp estão todos a $-3 \mathrm{~cm}$ e tanto o ponto $\mathrm{C}$ como o D estão dentro do intervalo - CVT $\mathrm{cm}$ e - (CVT 2) $\mathrm{cm}$; b- estádio I: o ponto de referência mais distal é $>1 \mathrm{~cm}$ acima do hímen; c- estádio II: o ponto de referência mais distal ao prolapso é $\leq 1$ $\mathrm{cm}$ proximal ou distal ao plano do hímen; d- estádio III: a porção mais distal do prolapso é $>1$ $\mathrm{cm}$ abaixo do hímen mas não mais que $2 \mathrm{~cm}$ menos que o CVT; e- estádio IV: completa eversão. A porção mais distal do prolapso está a pelo menos CVT $-2 \mathrm{~cm}$.

A análise estatística foi realizada a partir de tabelas de freqüências. A medida adotada para avaliar a concordância entre as duas terminologias é a estatística kappa. Para medidas com máximo grau de concordância ela assume o valor $2 \mathrm{e}$ para ausência de concordância, assume 0. Para valores da estatística inferiores a 0,4 , a concordância é considerada fraca ${ }^{11}$. Utilizamos para as análises o programa SAS 10.0.

O projeto foi aprovado pelo Comitê de Ética em Pesquisa da Universidade Federal de São Paulo/Escola Paulista de Medicina (Unifesp/EPM).

\section{Resultados}

Os achados ao exame físico das pacientes utilizando-se as terminologias de Baden e Walker e da ICS de acordo com o prolapso uterino, prolapso de parede vaginal anterior e de parede vaginal posterior podem ser observados, respectivamente, nas Tabelas 2, 3 e 4. Nota-se que em quase todas as pacientes, para cada estádio do prolapso genital, segundo a classificação da ICS, correspondem-se diferentes graus de prolapso do mesmo compartimento segundo Baden e Walker $^{7,8}$.

Observa-se somente para o prolapso de parede vaginal posterior estádio IV ( 1 paciente) e para o prolapso uterino estádio zero (29 pacientes) correspondência, segundo Baden e Walker, de 100\% com retocele grave e ausência de prolapso, respectivamente. 
Tabela 2 - Comparação entre as classificações de Baden e Walker ${ }^{7,8}$ e ICS ${ }^{2}$ para o prolapso uterino e de cúpula vaginal.

\begin{tabular}{lcccrrr}
\hline Baden e Walker,8 & Estádio 0 & Estádio I & $\begin{array}{c}\text { Classificação ICS } \\
\text { Estádio II }\end{array}$ & Estádio III & Estádio IV & Total \\
\hline Grau 0 & 29 & 40 & & & & 69 \\
Grau I & & 17 & 2 & 1 & 2 & 20 \\
Grau II & & 1 & 2 & 2 & 4 & 7 \\
Grau III & & & & 4 & 6 & 5 \\
Total & 29 & 58 & 4 & 4 & 101 \\
\hline
\end{tabular}

Estádio 0: $100 \%$ sem prolapso.

Estádio I: $68,9 \%$ sem prolapso, $29,3 \%$ prolapso de $1^{\circ} \mathrm{grau}, 1,8 \%$ prolapso de $2^{\circ} \mathrm{grau}$.

Estádio II: $50 \%$ prolapso de $1^{\circ}$ grau, $50 \%$ prolapso de $2^{\circ}$ grau.

Estádio III: $25 \%$ prolapso de $1^{\circ}$ grau, $50 \%$ prolapso de $2^{\circ}$ grau, $25 \%$ prolapso de $3^{\circ} \mathrm{grau}$.

Estádio IV: $33,3 \%$ prolapso de $2^{\circ}$ grau e $66,7 \%$ prolapso de $3^{\circ}$ grau.

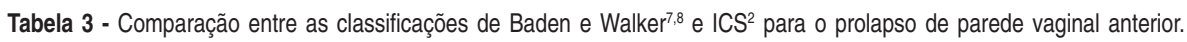

\begin{tabular}{|c|c|c|c|c|c|c|}
\hline \multirow[t]{2}{*}{ Baden e Walker ${ }^{7,8}$} & \multicolumn{5}{|c|}{ Classificação ICS² } & \multirow[b]{2}{*}{ Total } \\
\hline & Estádio 0 & Estádio I & Estádio II & Estádio III & Estádio IV & \\
\hline Grau 0 & 6 & 1 & & & & 7 \\
\hline Grau I & 1 & 11 & 24 & 1 & & 37 \\
\hline Grau II & & 1 & 35 & 1 & & 37 \\
\hline Grau III & & & 6 & 13 & 1 & 20 \\
\hline Total & 7 & 13 & 65 & 15 & 1 & 101 \\
\hline
\end{tabular}

Estádio 0: $85,7 \%$ sem prolapso, $14,3 \%$ prolapso de primeiro grau.

Estádio I: $7,6 \%$ sem prolapso, $84,8 \%$ prolapso de $1^{\circ}$ grau, $7,6 \%$ prolapso de $2^{\circ}$ grau.

Estádio II: $36,9 \%$ prolapso de $1^{\circ}$ grau, $53,8 \%$ prolapso de $2^{\circ}$ grau, $9,3 \%$ prolapso de $3^{\circ}$ grau.

Estádio III: $6,6 \%$ prolapso de $1^{\circ}$ grau, $6,6 \%$ prolapso de $2^{\circ}$ grau, $86,8 \%$ prolapso de $3^{\circ} \mathrm{grau}$.

Estádio IV: $100 \%$ prolapso de terceiro grau.

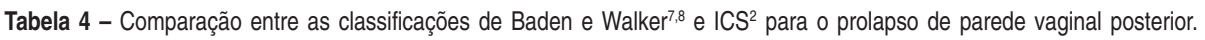

\begin{tabular}{|c|c|c|c|c|c|c|}
\hline \multirow[t]{2}{*}{ Baden e Walker ${ }^{7,8}$} & \multicolumn{5}{|c|}{ Classificação ICS² } & \multirow[b]{2}{*}{ Tota } \\
\hline & Estádio 0 & Estádio I & Estádio II & Estádio III & Estádio IV & \\
\hline Ausência de retocele & 12 & 3 & & & & 15 \\
\hline Retocele leve & 3 & 23 & 12 & & & 38 \\
\hline Retocele moderada & & 5 & 34 & 1 & & 40 \\
\hline Retocele grave & 2 & & 4 & 1 & 1 & 8 \\
\hline Total & 17 & 31 & 50 & 2 & 1 & 101 \\
\hline
\end{tabular}

Estádio 0: $70,5 \%$ sem prolapso, $17,6 \%$ prolapso de $1^{\circ}$ grau, $11,9 \%$ prolapso de $3^{\circ}$ grau. Estádio I: $9,6 \%$ sem prolapso, $74,2 \%$ prolapso de $1^{\circ} \mathrm{grau}, 16,2 \%$ prolapso de $2^{\circ}$ grau. Estádio II: $24 \%$ prolapso de $1^{\circ}$ grau, $68 \%$ prolapso de $2^{\circ}$ grau, $8 \%$ prolapso de $3^{\circ} \mathrm{grau}$. Estádio III: $50 \%$ prolapso de $2^{\circ}$ grau, $50 \%$ prolapso de $3^{\circ}$ grau.

Estádio IV: $100 \%$ prolapso de terceiro grau.

Para o prolapso uterino ou de cúpula vaginal observamos que das 58 pacientes classificadas como estádio I encontramos $40(68,9 \%)$ consideradas como ausência de prolapso, 17 como prolapso de $1^{\circ}$ grau e uma como prolapso de $2^{\circ}$ grau. Das 4 pacientes classificadas como estádio II, duas receberam o diagnóstico de prolapso de $1^{\circ}$ grau e duas de segundo grau. Das 4 pacientes consideradas estádio III, uma foi classificada como prolapso de $1^{\circ}$ grau, duas de $2^{\circ}$ grau e somente uma de $3^{\circ}$ grau. Por último, entre as 6 pacientes consideradas estádio IV, observamos duas como prolapso de $2^{\circ}$ grau e $4 \mathrm{com}$ prolaso de $3^{\circ}$ grau (Tabela 2 ).

$\mathrm{Na}$ utilização da medida do ponto $\mathrm{C}$ para quantificar o prolapso uterino, observamos que entre as pacientes consideradas sem prolapso por 
Baden e Walker ${ }^{7,8}$ o ponto $\mathrm{C}$ variou de $-10 \mathrm{a}-2 \mathrm{~cm}$. Naquelas consideradas com prolapso de $1^{\circ}$ grau variou de $-5 \mathrm{a}+3 \mathrm{~cm}$, nas com prolapso de $2^{\circ}$ grau variou de $-2 \mathrm{a}+6 \mathrm{~cm}$ e por último naquelas com prolapso de $3^{\circ}$ grau a medida variou de de $+5 \mathrm{a}+8$ cm (Tabela 5).

Tabela 5 - Variação em centímetros dos pontos definidos pela ICS: Ba, Bp e C, segundo a classificação de Baden e Walker 7,8 para o prolapso genital.

\begin{tabular}{lcccr}
\hline Pontos definidos & \multicolumn{4}{c}{ Baden e Walker,8 } \\
& Grau 0 & Grau I & Grau II & Grau III \\
\hline Ba & $-3 a+2$ & $-2 a+1$ & $-2 a+3$ & $+1 a+8$ \\
Bp & $-3 a+2$ & $-3 a 0$ & $-2 a+2$ & $0 a+8$ \\
C & $-10 a-2$ & $-5 a+3$ & $-2 a+6$ & $+5 a+8$ \\
\hline
\end{tabular}

Quanto ao prolapso de parede vaginal anterior, das 7 pacientes classificadas como estádio 0 encontramos 6 consideradas com ausência de cistocele e uma com cistocele de $1^{\circ}$ grau. Das 13 pacientes classificadas como estádio I, uma não apresentava cistocele, 11 tinham cistocele de $1^{\circ}$ grau e uma de $2^{\circ}$ grau. Das 65 pacientes consideradas estádio II, 24 apresentavam cistocele de $1^{\circ}$ o grau, 35 de $2^{\circ}$ grau e seis de $3^{\circ}$ grau. Das 15 pacientes com estádio III, uma era considerada cistocele de $1^{\circ}$ grau, uma cistocele de $2^{\circ}$ grau e 13 de $3^{\circ}$ grau. Por último, a única paciente considerada estádio IV seria classificada como cistocele de $3^{\circ}$ grau (Tabela 3 ).

Em relação à medida do ponto $\mathrm{Ba}$ para a quantificação do prolapso de parede vaginal anterior observamos que, no grupo de pacientes consideradas sem cistocele por Baden e Walker, este variava de $-3 \mathrm{a}+2 \mathrm{~cm}$. Naquelas consideradas com cistocele de $1^{\circ}$ grau variou de $-2 \mathrm{a}+1 \mathrm{~cm}$, nas com cistocele de $2^{\circ}$ grau variou de $-2 \mathrm{a}+3 \mathrm{~cm}$ e por último entre aquelas com cistocele de $3^{\circ}$ grau variou de $+1 \mathrm{a}+8 \mathrm{~cm}$ (Tabela 5).

Para o prolapso de parede vaginal posterior: no estádio zero estavam 17 pacientes, das quais 12 com ausência de retocele, três consideradas com retocele leve e 2 com retocele severa segundo Baden e Walker ${ }^{7,8}$. Entre as 31 pacientes classificadas como estádio I encontramos três consideradas com ausência de retocele, 23 com retocele leve e 5 com retocele moderada. Das 50 pacientes classificadas como estádio II, 12 eram classificadas com retocele leve, 34 retocele moderada e 4 retocele grave. Entre as 2 pacientes consideradas estádio III, uma tinha retocele moderada e a outra retocele grave. Por último, a única paciente considerada estádio IV também foi considerada como tendo retocele grave (Tabela 4).
Ao utilizarmos a medida do ponto Bp para a quantificação do prolapso de parede vaginal posterior, observamos que, nas pacientes consideradas sem retocele por Baden e Walker ${ }^{7,8}$, este variava $\mathrm{de}-3 \mathrm{a}+2 \mathrm{~cm}$. Naquelas consideradas com retocele leve, esta medida variou de -3 a $0 \mathrm{~cm}$, nas com retocele moderada variou de $-2 \mathrm{a}+2 \mathrm{~cm}$ e por último naquelas com retocele grave variou de $0 \mathrm{a}+8$ cm (Tabela 5).

Os valores da estatística kappa, que mede a concordância entre as duas classificações para os prolapsos uterino ou de cúpula vaginal, parede vaginal anterior e parede vaginal posterior, são respectivamente: 0,$312 ; 0,124$ e 0,225. Para os três tipos de prolapsos examinados, os valores estão abaixo de 0,4 , indicando fraca concordância entre as classificações.

\section{Discussão}

A classificação do prolapso pélvico feminino apresenta ampla variabilidade na sua padronização. Constitui o obstáculo mais importante encontrado para realização de pesquisa de alto nível de evidência científica. Muitos relatos usam termos que são indefinidos, não permitindo, por exemplo, comparações de estudos publicados entre diferentes instituições, nem mesmo avaliações longitudinais de uma paciente individualmente.

A classificação de Baden e Walker modificada por Juma et al. ${ }^{8}$, em 1993, é a de uso mais difundido em nosso meio. Contudo, a utilização de termos imprecisos como terço inferior da vagina, além da realização do exame com tração, certamente superestima os achados.

A ICS (International Continence Society) vem tentando padronizar a terminologia da disfunção do trato urinário baixo desde o estabelecimento de seu Comitê de Padronização de Terminologia. Os esforços durante as duas últimas décadas resultaram em aceitação mundial de padrões de terminologia que permitem aos pesquisadores comunicarem-se de modo eficiente. Desde 1996, após vários estudos multicêntricos para avaliação de sua reprodutibilidade ${ }^{12-14}$, essa nova terminologia foi adotada formalmente pela ICS, pela Sociedade Americana de Uroginecologia e pela Sociedade dos Cirurgiões Ginecologistas ${ }^{12}$.

A reprodutibilidade inter e intra-observador da classificação adotada pela ICS foi objeto de estudo de Hall et al. ${ }^{12}$. Estes autores encontraram que os estádios foram altamente reprodutiveis. Em nenhum caso houve variação maior que um estádio e em $69 \%$ dos casos o estadiamento realizado 
pelos dois observadores foi idêntico. Da mesma forma, houve forte correlação intra-observador, com $64 \%$ de coincidência nos estadiamentos.

Kobak et al. ${ }^{14}$, estudando 49 mulheres com incontinência urinária e/ou prolapso genital e que submeteram-se a exame clínico por dois observadores conforme preconiza a ICS, encontraram kappa de 0,79 (p<0,001), mostrando alta correlação inter-observador.

Dados recentes de estudo da UNIFESP/EPM demonstraram substancial reprodutibilidade interobservador na quantificação das medidas dos nove pontos específicos, encontrando correlação altamente substancial e significante. Da mesma forma a determinação do estádio do prolapso pélvico também foi altamente reprodutivel ${ }^{15}$. Entretanto, até o momento, em nosso meio, nenhum estudo havia confrontado as duas terminologias.

Em nosso estudo, pacientes com disfunção do assoalho pélvico submeteram-se à avaliação do prolapso genital pelo mesmo observador. Os examinadores já se utilizavam da terminologia da ICS nos seus exames cotidianos, pois o mesmo já é rotineiro em nosso setor. Inicialmente o observador realizava a avaliação segundo os critérios de Baden e Walker ${ }^{7,8}$ e a seguir os da ICS ${ }^{2}$.

Observamos sensiveis diferenças entre as aferições das terminologias. A utilização dos valores numéricos precisos com o intuito de aferir a intensidade do prolapso pela terminologia da $\mathrm{ICS}^{2}$ confronta-se claramente com a subjetividade da classificação de Baden e Walker ${ }^{7,8}$. Cada "grau" de prolapso, independente da sua topografia, correspondia a uma ampla variação de medidas numéricas em centímetros, denotando a maior precisão da classificação por estádios da $\mathrm{ICS}^{2}$. Naquelas pacientes consideradas com retocele grave, por exemplo, a porção mais dital da parede vaginal posterior (ponto $\mathrm{Bp}$ ) variou de $0 \mathrm{~cm} \mathrm{a}+8$ $\mathrm{cm}$. Naquelas com cistocele de $3^{\circ}$ grau a porção mais distal da parede vaginal anterior (ponto $\mathrm{Ba}$ ) variou de $+1 \mathrm{~cm} \mathrm{a}+8 \mathrm{~cm}$.

Em relação ao prolapso uterino, observamos que pacientes classificadas como prolapso de $1^{\circ}$ o grau foram aferidas como prolapso estádio I, II e III. Já aquelas classificadas como de $2^{\circ}$ grau variavam desde o estádio I até o IV da ICS ${ }^{2}$. Esses dados nos confirmam que a tração do colo uterino utilizado por Baden e Walker ${ }^{7,8}$ realmente pode superestimar as medidas naquelas pacientes com prolapso uterinos de menor intensidade.

Essas discrepâncias somente reforçam a convicção de que um sistema padronizado de avaliação do prolapso genital é necessário. Para tanto, a Sociedade Internacional de Continência, a Sociedade Americana de Uroginecologia e a Sociedade dos Cirurgiões Ginecologistas ${ }^{12}$ estabeleceram essa nova terminologia como padrão da avaliação da disfunção de assoalho pélvico, orientando seu uso rotineiro para todas as publicações internacionais. Preconiza-se, portanto, que os dados sejam registrados utilizando-se essa mesma terminologia padronizada, visando excluir discrepâncias entre as medidas, possibilitando avaliar de maneira mais fidedigna as variações nos resultados de intervenções terapêuticas em diferentes centros.

\section{ABSTRACT}

Objective: to compare Baden and Walker's (BW) classification system to the International Continence Society (ICS) standardization of terminology of female pelvic organ prolapse.

Methods: information about urogynecological investigation on 101 women, performed by the Urogynecology and Vaginal Surgery Sector of UNIFESP/EPM, was retrospectively analyzed. Only patients who had undergone the standard ICS exam which quantifies the pelvic prolapse were selected. According to ICS, the prolapse is analyzed through a standard reference system relating the hymen to the anatomic position of six vaginal points: two in the anterior vaginal wall, two in the vaginal apex and other two in the posterior vaginal wall. The maximum amount of pelvic organ prolapse was viewed and recorded during a Valsalva's maneuver. The measurement of the most distal point of the prolapse was performed and it was compared to the $B W$ classification system. The data were analyzed by kappa statistics, to assess the concordance between the two terminologies.

Results: There was total correspondence only for the posterior vaginal prolapse stage IV (one patient) and for the uterus prolapse stage 0 (29 patients) with severe rectocele and absence of prolapse, respectively, according to $B W$. In the three types of prolapses evaluated, the values of kappa statistics were below 0.4, indicating a weak concordance between the two terminologies. There is an extensive variation in the measurement of the most distal point of prolapse when the $B W$ classification is perfomed.

Conclusions: there is a weak concordance between the $B W$ classification system and the ICS standardization of terminology of female pelvic organ prolapse.

KEYWORDS: Genital prolapse. Pelvic floor. Stress urinary incontinence.

\section{Referências}

1. Gonçalves JG, Oliveira LM, Freitas MMS, Baracat EC. Prolapso genital. In: Girão MJBC, Rodrigues de Lima G, Baracat EC, editores. Uroginecologia. $1^{\text {a }} \mathrm{ed}$. São Paulo: Artes Médicas; 1997. p. 143-9. 
2. International Continence Society, Committe on Standardisation of Terminology. The standardization of terminology of lower urinary tract function. In: Ostergard DR, Bent AE, editors. Urogynecology and Urodynamics: theory and practice. $3^{\text {rd }}$ ed. Baltimore: Williams \& Wilkins; 1991. p. 545-62.

3. Kjerulff KH, Erickson BA, Langenberg PW. Chronic gynecological conditions reported by US women: findings from the National Health Interview Survey, 1984-1992. Am J Public Health 1996; 86:195-9.

4. Mallet VT, Bump RC. The epidemiology of female pelvic floor disfunction. Curr Opin Obstet Gynecol 1994; 6:308-12.

5. Ellerkmann RM, Cundiff GW, Melick CF, Nihira MA, Leffler K, Bent AE. Correlation of symptoms with location and severity of pelvic organ prolapse. Am J Obstet Gynecol 2001; 185:1332-7.

6. Swift SE, Pound T, Dias JK. Case-control study of etiologic factors in the development of severe pelvic organ prolapse. Int Urogynecol $\mathrm{J}$ Pelvic Floor Dysfunc 2001; 12:187-92.

7. Baden WF, Walker TA, Lindsey JH. The vaginal profile. Tex Med 1968; 64:56-8.

8. Juma S, Little NA, Raz S. Evaluation of stress urinary incontinence. In: Buchsbaum HJ, Schmidt JD, editors. Gynecologic and Obstetric Urology. $3^{\text {rd }}$ ed. Philadelphia: W.B. Saunders; 1993. p. 251-63.

9. Bump RC, Mattiasson A, Bo K, et al. The standardization of terminology of female pelvic organ prolapse and pelvic floor dysfunction. Am J Obstet Gynecol 1996; 175:10-7.
10.Weber AM, Abrams P, Brubaker L, et al. The standardization of terminology for researchers in female pelvic floor disorders. Int Urogynecol J Pelvic Floor Dysfunc 2001; 12:178-86.

11.Agresti A. An introduction to categorical data analysis. $1^{\text {st }}$ ed. New York: John Wiley and Sons; 1996.

12.Hall AF, Theofrastous JP, Cundiff GW, et al. Interobserver and intraobserver reliability of the proposed International Continence Society, Society of Gynecologic Surgeons, and American Urogynecologic Society pelvic organ prolapse classification system. Am J Obstet Gynecol 1996; 175:1467-71.

13.Schussler B, Peschers U. Standardization of terminology of female genital prolapse according to the new ICS criteria: interexaminer reproducibility. Neurourol Urodyn 1995; 14:437-8.

14.Kobak WH, Rosenberger K, Walters MD. Interobserver variation in the assessment of pelvic organ prolapse. Int Urogynecol J Pelvic Floor Dysfunct 1996; 7:121-4.

15.Feldner Júnior PC, Bezerra LRPS, et al. Reprodutibilidade interobservador da classificação da distopia genital proposta pela Sociedade Internacional de Continência. Rev Bras Ginecol Obstet 2003; 25:353-8. 